\title{
On-sky MOAO performance evaluation of RAVEN
}

\author{
Y. H. Ono ${ }^{a}$, Carlos M. Correia ${ }^{a}$, O. Lardière ${ }^{b}$, D. R. Andersen ${ }^{b}$, S. Oya ${ }^{c}$, M. Akiyama $^{d}$, D. \\ Gamroth $^{e}$, K. Jackson ${ }^{f}$, O. Martin ${ }^{a}$, and C. Bradley ${ }^{e}$ \\ ${ }^{a}$ Aix Marseille Université, CNRS, LAM UMR 7326, 13388 Marseille, France; \\ ${ }^{b}$ NRC-Herzberg, 5071 West Saanich Rd., Victoria, British Columbia, Canada; \\ ${ }^{c}$ TMT-J Project Office, NAOJ, 2-21-1 Osawa, Mitaka, Tokyo 181-8588, Japan; \\ ${ }^{d}$ Astronomical Institute, Tohoku University, 6-3 Aramaki, Aoba-ku, Sendai 980-8578, Japan; \\ ${ }^{e}$ Adaptive Optics Laboratory, University of Victoria, 3800 Finnerty Rd., Victoria V8P 5C2, \\ British Columbia, Canada; \\ ${ }^{f}$ Division of Engineering and Applied Science, California Institute of Technology, 1200 E. \\ California Boulevard MC 155-44, Pasadena, CA 91125, USA;
}

\begin{abstract}
This paper presents the AO performance we got on-sky with RAVEN, a Multi-Object Adaptive Optics (MOAO) technical and science demonstrator installed and tested at the Subaru telescope. We report Ensquared-Energy (EE) and Full Width at Half Maximum (FWHM) measured from science images on Subaru's IRCS taken during all of the on-sky observing runs. We show these metrics as function of different AO modes and atmospheric conditions for two asterisms of natural guide stars. The performances of the MOAO and Ground-Layer AO (GLAO) modes are between the classical Single-Conjugate AO (SCAO) and seeing-limited modes. We achieve the $\mathrm{EE}$ of $30 \%$ in $\mathrm{H}$-band with the MOAO correction, which is a science requirement for RAVEN. The MOAO provides sightly better performance than the GLAO mode in both asterisms. One of the reasons which cause this small difference between the MOAO and GLAO modes may be the strong GL contribution. Also, the performance of the MOAO modes is affected by the accuracy of the on-sky turbulence profiling by the SLOpe Detection And Ranging (SLODAR) method.
\end{abstract}

Keywords: adaptive optics, multi-object adaptive optics, RAVEN

\section{INTRODUCTION}

Multi-Object Adaptive Optics (MOAO) is one of wide-filed adaptive optics systems, which can apply AO correction simultaneously to multiple science objects over a large Field of Regard (FoR), and improve the multiplicity and efficiency of AO-assisted observations. ${ }^{1}$ The instruments of multiple Integral Field Spectrographs (IFSs) with MOAO corrections are planed for future Extreme Large Telescopes (ELTs), ${ }^{2-4}$ and these instruments will have 10 20 multiplicities over a FoR of $5 \sim 10$ arcmin diameter. However, there are some technical difficulties associated with MOAO systems: alignment, calibration and tomographic wavefront reconstruction on the openloop control. Therefore, a few technical demonstrators ${ }^{5-7}$ have been developed and tested on-sky to mitigate these technical risks before building future facility-class MOAO instruments.

The RAVEN project ${ }^{8}$ is the first MOAO science and technical demonstrator on an $8 \mathrm{~m}$ class telescope, which was installed at the Subaru telescope at Maunakea in Hawaii. Raven is developed by the University of Victoria (UVic) in partnership with the National Research Council of Canada (NRC), the National Astronomical Observatory of Japan (NAOJ) and Tohoku university. Fig.1 shows the functional optical block diagram of the RAVEN system. RAVEN performs the tomographic wavefront reconstruction with three or four open-loop ShackHartmann WFSs (SH-WFSs) to reconstruct the atmospheric turbulence volume above the telescope. Then, RAVEN applies MOAO corrections simultaneously to two science objects over a FoR of 2 arcmin diameter by a Deformable Mirrors (DM) with $11 \times 11$ actuators installed in each science optical path. RAVEN has also a closedloop SH-WFS in each science optical path for calibration, classical closed-loop Single-Conjugate AO (SCAO)

Further author information: yoshito.ono@lam.fr

Adaptive Optics Systems V, edited by Enrico Marchetti, Laird M. Close, Jean-Pierre Véran, Proc. of SPIE Vol. 9909,990910 (C) 2016 SPIE · CCC code: 0277-786X/16/\$18 · doi: 10.1117/12.2232321 


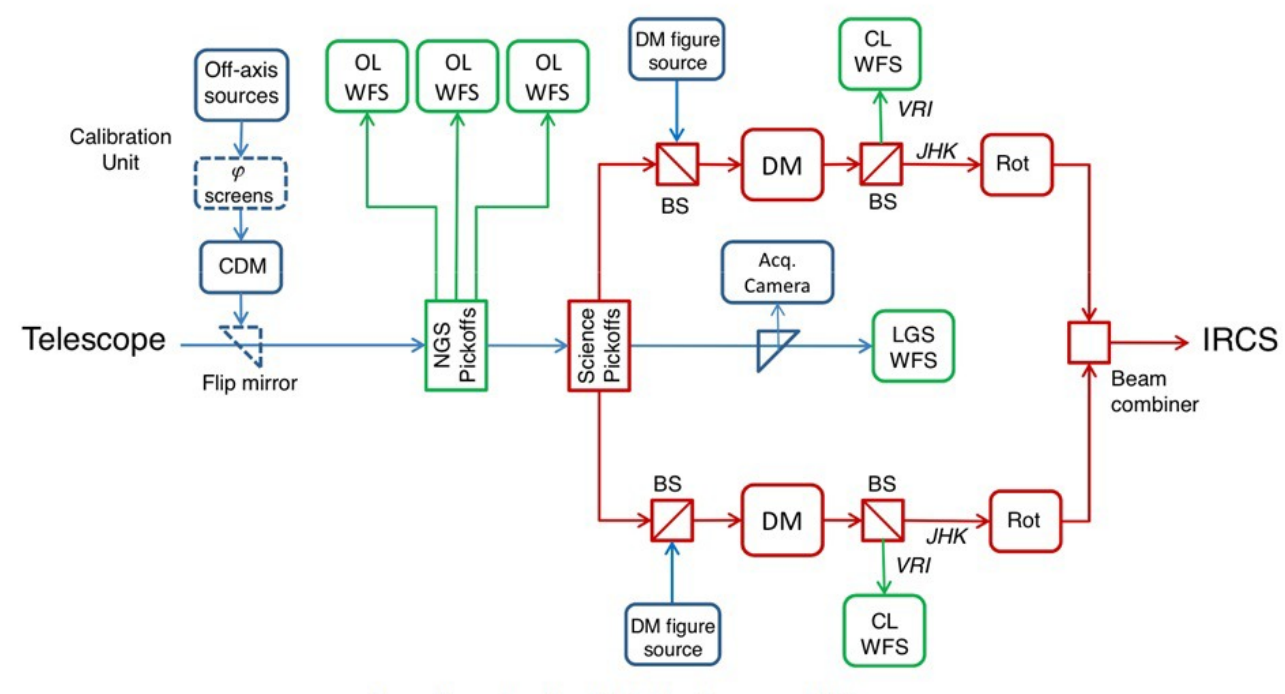

Functional optical block diagram of Raven.

Figure 1. Block diagram of RAVEN (the image from http://web.uvic.ca/).

correction and measuring the phase distortion in the science direction. Each SH-WFS has $10 \times 10$ subapertures, and the subaperture size corresponds to $0.8 \mathrm{~m}$ on the pupil plane. The corrected science lights are aligned and delivered to the IRCS,$^{9}$ which is a near-infrared imaging spectrograph in the Subaru telescope. RAVEN also can do the SCAO and open-loop ground layer AO (GLAO) correction by averaging the measurement over all open-loop SH-WFSs. The detailed RAVEN design is summarized in Lardière et al. (2014). ${ }^{8}$

We have successfully completed the three on-sky runs on the Subaru telescope with RAVEN, and the total number of night is 12 nights: The first run in May 13-14, 2014, the second run in August 9-10, 2014, and the third run in und 23-July 1, 2015. The very first result based on the result from the first run has already been reported in Lardière et al. (2014). ${ }^{8}$ In this paper, we present the statistical on-sky performance of RAVEN using the IRCS images and the WFS telemetries from the all three on-sky runs. In Section 2, we briefly describe our asterisms used on-sky and focused in this paper. Then, we explain our turbulence profiling method, which is required to optimize the MOAO performance, in Section 3, and, in Section 4, present the on-sky AO performance with RAVEN in terms of Full Width at Half Maximum (FWHM) and Ensquared Energy (EE) in a 140 mas box, which is a width of one of the IRCS slits. Finally, we conclude our results in Section 5.

\section{ASTERISM}

We focus in this paper on two asterisms of NGSs shown in Fig.2. The asterism 1 is one of our major engineering field, and we observe this field mostly at the beginning of the night in the first and third run. The averaged angular distance of NGSs from the center of the field is roughly 55 arcsec and the brightness of the NGSs are 10.2-12.5 mag in R-band. The limiting magnitude of RAVEN for the NGS is roughly 14 mag in R-band. The target stars are located close to the center of the field. The asterism 2 is observed in the second half of the night on June 28th in the third run. This field has larger angular separations of NGSs than that of asterism 1. In the asterism 2, there are many stars in the field, that can be used for a target, and we test the AO corrections with RAVEN in several different directions over the field. The NGSs in this field are brighter than $11.4 \mathrm{mag}$ in R-band.

\section{TURBULENCE PROFILING}

The turbulence profile, which is $C_{N}^{2}$ and the outer scale $\mathcal{L}_{0}$ at each altitude, is necessary for optimizing the tomographic reconstruction matrix. During the on-sky runs with RAVEN, we estimate the turbulence profile 
using SLOpe Detection And Ranging (SLODAR), ${ }^{10}$ which is a method to retrieve the turbulence profile from the measurements of multiple SH-WFSs. The SLODAR used in the on-sky runs does not estimate the outer scale and it was fixed to $30 \mathrm{~m}$ over all altitudes. However, the trends that the outer scale increases with the altitude are reported ${ }^{11}$ and, therefore, this assumption may have impacts on the turbulence profile estimation. Furthermore, the tomographic reconstruction matrix for the MOAO correction is created using the estimated turbulence profile. It means that the MOAO performance during the on-sky runs is not optimized. We improved the SLODAR method to estimate the outer scale and turbulences above the maximum altitude of the SLODAR. We reprocess the turbulence profiling offline for all on-sky telemetries using the improved SLODAR method. ${ }^{11}$ From the reprocessing with the offline SLODAR, the mean seeing over all nights is 0.475 arcsec, and $55 \%$ turbulence is in the Ground Layer (GL) (below $1.5 \mathrm{~km}$ ) on average over all nights. When we observed the asterism 1, there were a strong GL contribution and the median value of the $C_{N}^{2}$ fraction of the GL is $73 \%$. On the other hand, the GL $C_{N}^{2}$ fraction during the observation of the asterism 2 is lower than that during the observation of the asterism 1, and the median value is $53 \%$.

\section{ON-SKY PERFORMANCE}

In this section, we summarize statistical analysis on the PSF images obtained with IRCS during the on-sky observing runs. It should be noted that the results may be affected by the limitation of the SLODAR method used in the on-sky observations described above..

Fig.3 shows the on-sky FWHM (the top panel) and EE (the bottom panel) in H-band for the science channel 2 in the asterism 1 as a function of the seeing at $500 \mathrm{~nm}$ with different AO modes. The seeing at $500 \mathrm{~nm}$ is estimated by the off-line SLODAR. The median FWHM and EE in H-band with the MOAO and GLAO corrections are summarized in Table 1. The performances of the MOAO and GLAO modes are between the performances of the seeing-limited and SCAO modes as expected. We achieve the EE more than $30 \%$ in $\mathrm{H}$-band with the MOAO correction, which is a science requirement for RAVEN. Although there is no clear difference in FWHM and EE between the MOAO and GLAO modes in Fig.3, Table 1 shows that the median values of the FWHM and EE with MOAO mode are slightly better than those with the GLAO mode.

One of the reasons causing this small performance difference between the MOAO and GLAO corrections is a large GL contribution during the observation of the asterism 1. The median GL fraction is $73 \%$, and, therefore, most of turbulence can be corrected by the GLAO mode and the advantage of the MOAO mode gets small. Fig. 3 shows that we can achieve the FWHM less than 0.2 arcsec with the GLAO correction over a wide range of seeing at $500 \mathrm{~nm}$. Another reason would be the impact of the turbulence profiling. As mentioned before, the on-sky SLODAR assumes the same outer scale over all altitudes, and it may affects the estimation of the turbulence profile and the tomographic reconstruction. Indeed, we find some discrepancies between the profiles
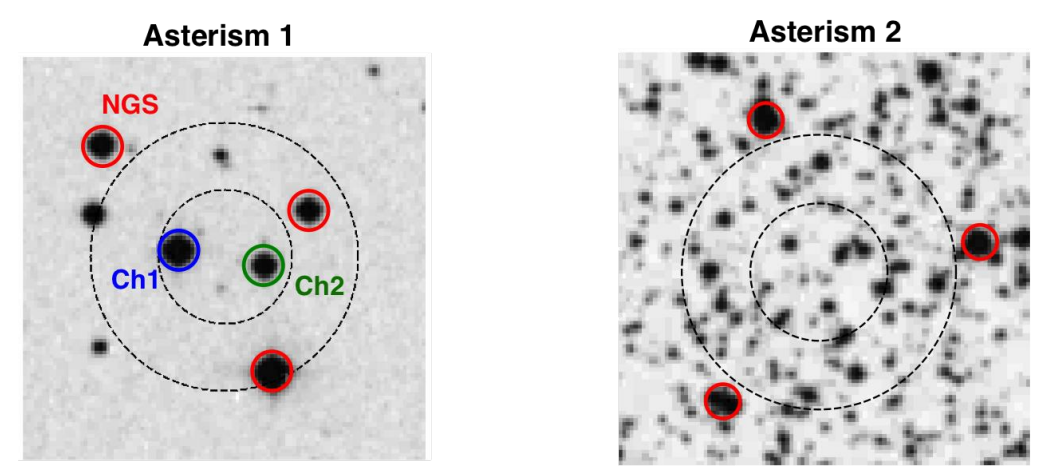

Figure 2. Asterisms observed during the on-sky observations. Left : Asterism 1. The right ascension and declination of the center of the astersim 1 are 15:15:31 and +05:15:59, respectively. The averaged distance of NGSs from the center is $\sim 55$ arcsec. The NGS brightnesses are 10.2-12.5 mag in R-band. Right : Asterism 2. The right ascension and declination of the center of the astersim 2 are 19:45:26 and +33:25:32, respectively. The averaged distance of NGSs from the center is $\sim 70$,arcsec. The NGS brightnesses are 10.6-11.4 mag in R-band. 

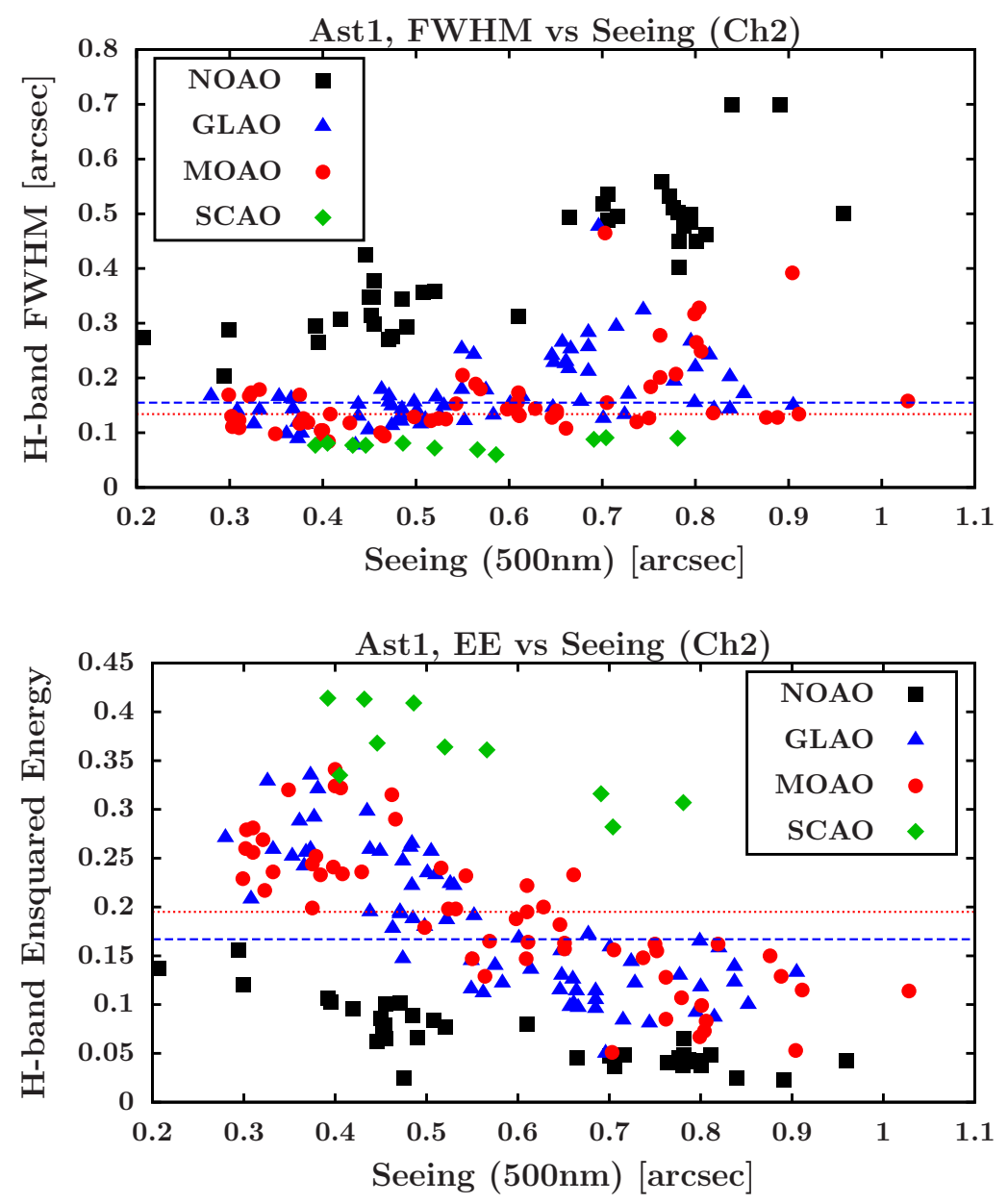

Figure 3. On-sky FWHM (top) and EE (bottom) in H-band for the science channel 2 in the asterism 1 as a function of the seeing at $500 \mathrm{~nm}$. The different symbols show the performances with different AO modes: no AO correction (black squares), SCAO (green diamonds), GLAO (blue triangles) and MOAO (red circles). The horizontal dashed and dotted lines represents the median values for GLAO and MOAO, respectively, which are summarized in Table 1

\begin{tabular}{ccccc}
\hline & \multicolumn{2}{c}{ FWHM $[\operatorname{arcsec}]$} & \multicolumn{2}{c}{ EE } \\
\cline { 2 - 5 } & GLAO & MOAO & GLAO & MOAO \\
\hline \hline Ch1 & 0.166 & 0.154 & 0.144 & 0.175 \\
Ch2 & 0.155 & 0.134 & 0.167 & 0.195 \\
\hline
\end{tabular}

Table 1. The median FWHM and EE with MOAO and GLAO corrections for the asterism 1.

estimated by the on-sky and offline SLODAR. In order to clarify the effect of the estimated turbulence profile, it is important to compute the tomographic error by "reply-mode" using on-sky WFS telemetries.

As shown in Table 1, the performance of the science channel 2 is better than the performance of the channel 1. This is because the position of the target picked-off by the science channel 2 is closer to the two NGSs than the position of the science target 1, and, therefore, the science channel 2 has less tomographic error than the channel 1.

Fig.4 presents the on-sky performance of the MOAO and GLAO modes for the science channel 1 in the asterism 2. These panels show that the MOAO performance is better than the GLAO performance, and it is because the GL $C_{N}^{2}$ fraction is lower than the case of the asterism 1 and the MOAO has more advantage for high 

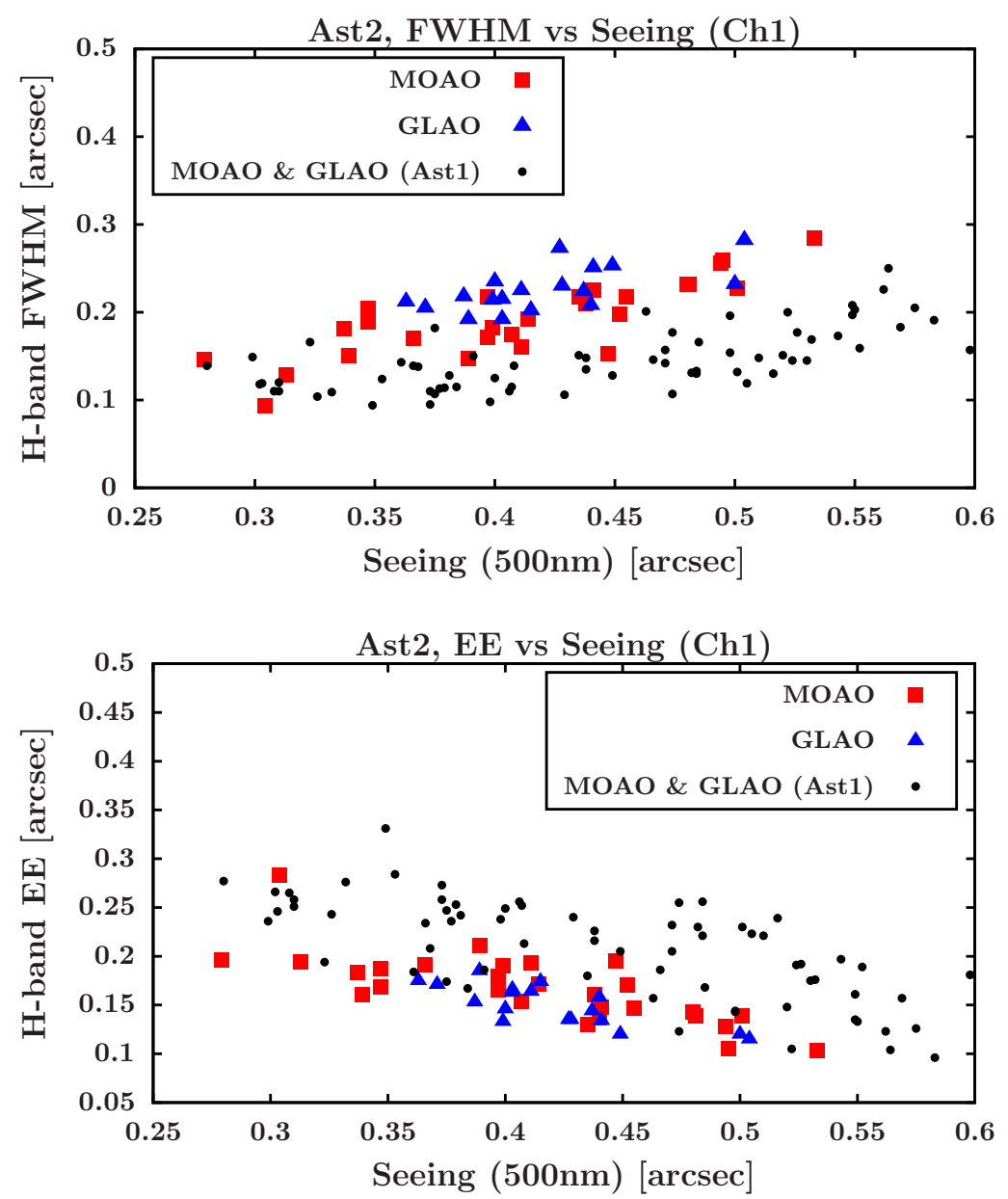

Figure 4. On-sky FWHM (top) and EE (bottom) in H-band for the science channel 1 in the asterism 2 as a function of the seeing at $500 \mathrm{~nm}$. The different symbols show the performances with different AO modes: GLAO (blue triangles) and MOAO (red circles). The black points represent the performance with GLAO and MOAO corrections in the asterism 1, which are plotted in Fig.3

altitude layers compared to the GLAO mode. The black dots in Fig.4 show the performance of the MOAO an GLAO modes for the asterism 1. The performance for the asterism 2 is worse than the values for the asterism 1 because of the larger tomographic error due to the wide separation between the NGSs.

\section{CONCLUSION}

RAVEN is a MOAO technical and science demonstrator on the Subaru telescope. We have successfully completed all of the on-sky observing runs with RAVEN and the Subaru telescope. In this paper, we present the AO performance we got on-sky with RAVEN. The AO performance is evaluated with FWHM and EE in a 140 mas box measured from the on-sky IRCS images in H-band. We focus on the two asterisms. In the asterism 1, the FWHM and EE with the MOAO and GLAO modes are between those of the SCAO and seeing-limited modes. Although the MOAO mode provides better performance than the GLAO modes, the difference in the performance between the MOAO and GLAO corrections is small. The one of the reasons causing this small difference may be the strong GL contribution, which the median GL fraction is $73 \%$ when we observe the asterism 1 . Since the SLODAR method used on-sky assumes the same outer scale over all altitudes, this also affects the MOAO performance. The GLAO mode provides the FWHM less than 0.2 arcsec in H-band over a wide range of seeing from 0.3 to 0.8 in the asterism 1. This result encourages the plans of future GLAO systems at Maunakea. In 
the case of the asterism 2, the advantage of the MOAO modes can be seen more clearly than the case of the asterism 1 because the GL $C_{N}^{2}$ fraction during the observation of the asterism 2 is less than that of the asterism 1. In order to understand the RAVEN on-sky performance, it is needed to make error breakdown to identify the error source and comparison with the numerical simulation.

\section{Acknowledgement}

The research leading to these results received the support of the JSPS Grant-in-Aid for JSPS Fellows (15J02510), the JSPS Grant-in-Aid for Young Scientist (B) (23740140), the JSPS Grant-in-Aid for Scientific Research (B) (26287027) and the $\mathrm{A}^{*}$ MIDEX project (no. ANR-11-IDEX-0001- 02) funded by the Investissements d'Avenir French Government program, managed by the French National Research Agency (ANR).

\section{REFERENCES}

[1] Hammer, F. et al., "The FALCON concept: multi-object spectroscopy combined with MCAO in near-IR," Proc. ESO workshop, 139-148 (2002).

[2] Andersen, D. R. et al., "The MOAO system of the IRMOS near-infrared multi-object spectrograph for TMT," Proc. SPIE 6269, 62694K-62694K-12 (2006).

[3] Akiyama, M. et al., "TMT-AGE: wide field of regard multi-object adaptive optics for TMT," Proc. SPIE 9148, 914814-914814-14 (2014).

[4] Hammer, F. et al., "MOSAIC at the E-ELT: A multi-object spectrograph for astrophysics, IGM and cosmology," Proc. SPIE 9147, 914727-914727-7 (2014).

[5] Gavel, D. et al., "Visible light laser guidestar experimental system (Villages): on-sky tests of new technologies for visible wavelength all-sky coverage adaptive optics systems," Proc. SPIE 7015, 70150G-70150G-11 (2008).

[6] Andersen, D. R. et al., "VOLT: the Victoria Open Loop Testbed," Proc. SPIE 7015, 70150H-70150H-11 (2008).

[7] Vidal, Fabrice et al., "Analysis of on-sky MOAO performance of CANARY using natural guide stars," $A \mathscr{E} A$ 569, A16 (2014).

[8] Lardière, O. et al., "Multi-object adaptive optics on-sky results with Raven," Proc. SPIE 9148, 9148-14 (2014).

[9] Tokunaga, A. T. et al., "Infrared camera and spectrograph for the subaru telescope," Proc. SPIE 3354, 512-524 (1998).

[10] Wilson, R. W., "SLODAR: measuring optical turbulence altitude with a ShackHartmann wavefront sensor," Monthly Notices of the Royal Astronomical Society 337(1), 103-108 (2002).

[11] Ono, Y. H. et al., "The statistics of atmospheric turbulence at Maunkea measured by RAVEN," these proceeding , 9909-138 (2016).

[12] Hayano, Y. et al., "UlTIMATE-SUBARU: project status," Proc. SPIE 9148, 91482S-91482S-8 (2014).

[13] Chun, M. R. et al., "imaka: ground-layer AO at Maunakea," these proceeding , 9909-2 (2016). 